\title{
Dynamical structure functions from quantum Monte Carlo calculations of a proper integral transform
}

\author{
Alessandro Roggero, ${ }^{*}$ Francesco Pederiva, ${ }^{\dagger}$ and Giuseppina Orlandini ${ }^{\ddagger}$ \\ Dipartimento di Fisica, Università di Trento, via Sommarive, 14 I-38123 Trento, Italy \\ and INFN - TIFPA, Trento Institute for Fundamental Physics and Applications, Trento, Italy
}

(Received 25 September 2012; revised manuscript received 22 January 2013; published 13 September 2013)

\begin{abstract}
An $a b$ initio method for determining the dynamical structure function of an interacting many-body quantum system has been devised by combining a generalized integral transform method with quantum Monte Carlo (QMC) methods. A kernel has been found that (i) lets the transform be calculable with QMC methods and (ii) is a representation of the $\delta$ function, allowing an inversion of the transform with a much higher predictive power than the inverse Laplace transform. As a first application, the excitation spectrum of bulk atomic ${ }^{4} \mathrm{He}$ has been computed, both in the low and intermediate momentum ranges. The peculiar form of the kernel allows us to predict, without using any model, both positions and widths of the collective excitations in the maxon-roton region, as well as the existence of the second collective peak. A prediction of the dispersion of the single-particle modes described by the incoherent part is also presented.
\end{abstract}

DOI: 10.1103/PhysRevB.88.094302

PACS number(s): 67.25.D-, 02.70.Ss, 05.10.-a

\section{INTRODUCTION}

Many important physical properties of matter are closely related to the underlying microscopic dynamics. The computation of some quantities such as, e.g., viscosity or magnetic susceptibility, is routinely performed for classical systems. For quantum many-body systems, thanks to the growing computational capabilities, we currently have a number of methods capable of a true ab initio treatment of ground states, however, the detailed study of dynamical properties yet has been elusive.

In this paper, we will focus on the problem of extending a class of quantum Monte Carlo (QMC) algorithms to accurately determine the dynamical structure function (DSF) for a generic quantum many-body system and a generic excitation operator. Such an extension is based on the use of the integral transform (IT) technique with generalized kernels. The amount and quality of information that can be extracted by the proposed scheme is illustrated in the application to the study of the coherent and incoherent density excitation spectra in ${ }^{4} \mathrm{He}$.

At present, QMC calculations provide benchmark results for a wide variety of many-body systems, ranging from atoms to ultracold gases to nuclei. The most dramatic limitation of QMC methods is their inability to treat dynamical properties in a similarly reliable way. This failure is essentially due to the fact that QMC works in imaginary time rather than in real time. This implies that quantities that do not directly translate into imaginary-time language, when analytically continued to real time, are affected by statistical noise that can be hardly reduced, making calculations unfeasible.

The mainstream approach to the problem is to attempt a numerical inversion starting from the imaginary-time autocorrelation function, i.e., the Laplace transform of the DSF, which is an easily accessible quantity in most QMC calculations. As is well known, this is an ill-posed problem $^{1}$ and sophisticated regularization techniques ${ }^{2,3}$ are needed to correctly extract the physical information. These regularization procedures are fundamental in ill-posed problems as are the inversions of integral transforms, however, their importance is strictly connected to the "degree of ill-posedness" of the problem. The
Laplace transform case is one of the worst ones in this respect. We can have an intuitive feeling of the reason considering that, due to the infinite range of the kernel function, many details of the structure of the excitation spectrum are washed out in the transformed function and are difficult to recover by means of the inversion procedure. Consequently, a large amount of physical information is often required in the inversion process, limiting the predictive power of the approach.

One of the physical problems where many inversion schemes have been applied and continuously improved is the resolution of the measured double-peaked structure of $S(\mathbf{q}, \omega)$ in superfluid ${ }^{4} \mathrm{He},{ }^{4}$ corresponding to a higher-energy collective roton mode. One of the most powerful and popular inversion schemes, the maximum entropy method ${ }^{5,6}$ (MEM), can not resolve this double-peaked structure. Only the genetic inversion via falsification of theories ${ }^{7}$ (GIFT) finally has been able to reproduce qualitatively this double-peak structure. Other sophisticated techniques were developed over time, such as the average spectrum method ${ }^{8}$ (ASM) and the stochastic analytic continuation ${ }^{9}$ (SAC), but unfortunately there were no applications to this particular problem.

Up to now, almost all the research efforts have been devoted to improving inversion algorithms in order to alleviate the inevitable uncertainties connected to the particular regularization scheme used, but very little attention has been devoted to lowering the ill-posedness of the problem beforehand. This can be accomplished using kernel functions different from the Laplace one.

Following this idea, the problem of computing various charge and current DSF of few-nucleon systems has been solved by using a generalized integral transform approach, i.e., the Lorentz integral transform (LIT) method. ${ }^{10,11}$ The success of this approach is due to the specific choice of the Lorentzian function as a kernel of the IT. On the one hand, this choice allows us to calculate the transform with bound-state techniques, even in the continuum region, although avoiding its discretization. On the other hand, and most important, the fact that the kernel is a representation of the $\delta$ function allows for a reliable and stable inversion, which is predictive on the $\mathrm{DSF}$, at a controlled resolution. We will give some ideas later 
in the text on why this is the case. So far, the application of this technique has been limited to a small number of particles [up to $N=6$ (Ref. 12) and 7 (Ref. 13)], due to computational limits of the few-body bound-state techniques.

In the following, we will discuss how to extend the idea of the LIT method to many-body systems, by developing a QMC equivalent. This is a major change of perspective with respect to past work on DSF calculations with Monte Carlo methods, where the focus has always been on the improvement of the inversion algorithm used rather than alleviating a priori the ill-posedness of the problem by a wise choice of the transform to calculate.

\section{FORMALISM}

At zero temperature, the contribution to the response of a system of interacting particles, due to a perturbative probe which transfers momentum $\mathbf{q}$ and energy $\omega$ to it, can be expressed using a spectral representation

$$
\begin{aligned}
S_{\hat{O}}(\mathbf{q}, \omega) & =\sum_{\nu}\left|\left\langle\Psi_{\nu}|\hat{O}(\mathbf{q})| \Psi_{0}\right\rangle\right|^{2} \delta\left(E_{\nu}-\omega\right) \\
& =\left\langle\Psi_{0}\left|\hat{O}^{\dagger}(\mathbf{q}) \delta(\hat{H}-\omega) \hat{O}(\mathbf{q})\right| \Psi_{0}\right\rangle,
\end{aligned}
$$

where $\left|\Psi_{\nu}\right\rangle$ are the final states of the reaction with energy $E_{\nu}$, $\left|\Psi_{0}\right\rangle$ is the ground state of the system (with energy $E_{0}=0$ ), $\hat{O}$ is an excitation operator, and $\delta(\hat{H}-\omega)$ is the spectral density of the Hamiltonian. The summation is extended to all discrete and continuum spectrum states in the set.

The cost of a direct calculation of $S_{\hat{O}}(\mathbf{q}, \omega)$ becomes rapidly prohibitive, as the number of particles or the energy transfer $\omega$ increase. The latter problem gets even more difficult when the final state is in the continuum since one would need to solve the many-body scattering problem.

One can instead consider an IT of $S_{\hat{O}}(\mathbf{q}, \omega)$ with a generic kernel $K(\sigma, \omega)$ :

$$
\Phi(\mathbf{q}, \sigma)=\int K(\sigma, \omega) S_{\hat{O}}(\mathbf{q}, \omega) d \omega .
$$

The substitution of the expression (1) for $S_{\hat{O}}(\mathbf{q}, \omega)$ yields

$$
\begin{aligned}
\Phi(\mathbf{q}, \sigma) & =\sum_{\nu}\left\langle\Psi_{0}\left|\hat{O}^{\dagger}(\mathbf{q})\right| \Psi_{v}\right\rangle K(\sigma, \omega)\left\langle\Psi_{v}|\hat{O}(\mathbf{q})| \Psi_{0}\right\rangle \\
& =\left\langle\Psi_{0}\left|\hat{O}^{\dagger}(\mathbf{q}) K(\sigma, \hat{H}) \hat{O}(\mathbf{q})\right| \Psi_{0}\right\rangle .
\end{aligned}
$$

Equation (4) can be viewed as a generalized sum rule which depends on a continuous parameter $\sigma$. Provided that the kernel and the excitation operator have suitable analytic properties, the right-hand side of Eq. (4) can be expressed via the mean value of an operator on a ground state. However, in few cases the transform is actually calculable with bound-state techniques. In nuclear physics, typical few-body bound-state techniques have been used to calculate the transforms with both the Stieltjes kernel ${ }^{14}$ as well as for the Lorentz kernel. ${ }^{10}$ However, while in the former case the inversion of the transform, whose kernel has a similar form as the Laplace one, has been shown to be problematic, ${ }^{15}$ in the latter case, even a rather simple regularization procedure has allowed us to obtain accurate and stable results. ${ }^{11}$

The reason can be easily understood. In the case of the Stieltjes or of the Laplace kernel, the information about
$S_{\hat{O}}(\mathbf{q}, \omega)$ in the $\omega$ domain is spread in a large- $\sigma$ domain. On the contrary, the Lorentz kernel, as well as any function that is a $\delta$-function representation, keeps that information in an arbitrarily narrow- $\sigma$ domain, governed by the width of the kernel. (In the $\delta$-function limit of the kernel, no inversion would be needed!)

Very few $\delta$-function representations have a practical implementation. In the past, the use of Gaussian kernels has been investigated in different fields, from condensed matter ${ }^{16,17}$ to nonperturbative QCD,${ }^{18,19}$ with limited results. Here, the idea is to recast one possible $\delta$-function representation in the imaginary-time propagation language, typical of QMC methods, similarly to what was suggested in Ref. 20.

Consider the following family of integral kernels

$$
K_{P}(\sigma, \omega)=N\left[\frac{e^{-\mu \frac{\omega}{\sigma}}}{\sigma}-\frac{e^{-\nu \frac{\omega}{\sigma}}}{\sigma}\right]^{P},
$$

where

$$
\mu=\frac{\ln [b]-\ln [a]}{b-a} a ; \quad v=\frac{\ln [b]-\ln [a]}{b-a} b,
$$

and the parameters $P, a, b$ are integer numbers with $b>a$. The normalization constant $N$ is a function of $P, a, b$ such that $\int d \sigma K_{P}(\sigma, \omega)=1$.

The efficiency of this transform is due to the fact that $K_{P}(\sigma, \omega)$ converges to $\delta(\omega-\sigma)$ in the $P \rightarrow \infty$ limit, independent on the choice of $a$ and $b$. For a finite $P$ at each value of $\omega$ the kernel has a finite width that depends on $P$ and that represents a sort of resolution at which one can study the DSF. Moreover, $\sigma K_{P}(\sigma, \omega)$ has a maximum around $\omega=\sigma$. Therefore, the main advantage is that one can choose both the energy range of interest (the $\sigma$ values) and the resolution (larger values of $P$ correspond to higher resolution) for the study of the DSF. This makes the approach extremely flexible, similarly to the case of the LIT method.

Using a binomial expansion, and rewriting powers as exponential functions, leads to a form of the kernel that is a linear combination of the so-called Sumudu transform kernels, ${ }^{21}$ and is more transparent in view of a QMC calculation:

$$
K_{P}(\sigma, \omega)=\frac{N}{\sigma} \sum_{k=0}^{P}\left(\begin{array}{l}
P \\
k
\end{array}\right)(-1)^{k} e^{-\ln (b / a)\left[\frac{a}{b-a} P+k\right] \frac{\omega}{\sigma}} .
$$

In fact, by operating the substitution $\omega \rightarrow \hat{H}$ according to Eq. (4), we are led to a simple linear combination of imaginarytime propagators $(\hbar=1)$

$$
K_{P}(\sigma, \hat{H})=\frac{N}{\sigma} \sum_{k=0}^{P}\left(\begin{array}{l}
P \\
k
\end{array}\right)(-1)^{k} e^{-\tau_{P k} \hat{H}},
$$

taken at different imaginary-time points

$$
\tau_{P k}=\ln (b / a)\left[\frac{a}{b-a} P+k\right] / \sigma .
$$

\section{A. SVD analysis}

It is possible to characterize in a more quantitative way this kernel as compared to other choices by looking at the singular value expansion (SVE) of the kernel function or, better yet, at the singular value decomposition (SVD), since we are dealing 


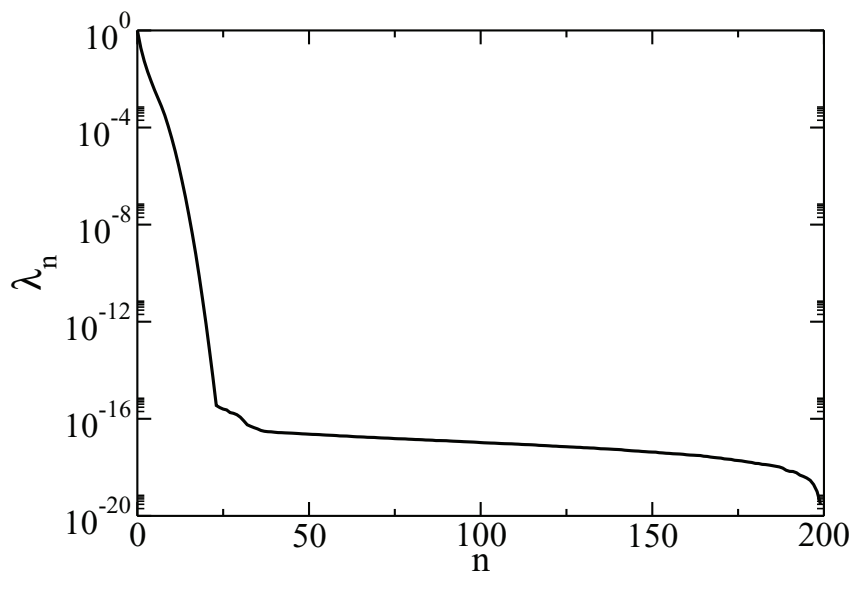

FIG. 1. Eigenvalues of the SVD for the Laplace kernel on an $N=200$ mesh-points grid. A $\delta$ function would give all eigenvalues equal to one.

with a discrete set of data. We start by discretizing the integral equation (3) on an $N$-point grid

$\Phi(\sigma)=\int K(\sigma, \omega) S(\omega) d \omega \rightarrow \Phi_{i}=\sum_{k}^{N} K_{i k} S_{k} \quad i \in[1, N]$,

where we have defined

$$
\Phi_{i} \equiv \Phi\left(\sigma_{i}\right), \quad K_{i k} \equiv \alpha_{k} K\left(\sigma_{i}, \omega_{k}\right), \quad S_{k} \equiv S\left(\omega_{k}\right),
$$

where $\alpha_{k}$ are the coefficients used for the particular integration scheme employed.

Now, we can apply the SVD to the kernel matrix $K$ to produce the following factorization:

$$
K=U \Lambda V^{T}, \quad U, V, \Lambda \in \mathbf{R}^{N x N}
$$

with $U, V$ orthogonal matrices and $\Lambda=\operatorname{diag}\left[\lambda_{1}, \ldots, \lambda_{N}\right]$. The positive-definite elements on the diagonal of $\Lambda$ are called singular values and can be organized in descending order: $\lambda_{1}>\lambda_{2}>\cdots>\lambda_{N}$, while the column vectors of $U$, and $V, \bar{u}_{i}$, and $\bar{v}_{i}$, are called left and right singular vectors, respectively. Usually, when dealing with integral transforms (Fredholm integral equation of the first kind), the rate of decay of singular values $\lambda_{n}$ is at least exponential in $n$ (e.g., see Fig. 1), and the singular vectors $\bar{u}_{i}$ and $\bar{v}_{i}$ can be viewed as discrete functions with an increasing number of sign changes. In the SVE limit, these would be functions with an increasing number of nodes, therefore corresponding to contributions with increasing frequencies.

Using this decomposition, we can obtain the following expressions for both the direct and inverse problems:

$$
\begin{gathered}
\bar{\Phi}=K \bar{S}=\sum_{j}^{N} \lambda_{j}\left(\bar{v}_{j}^{T} \bar{S}\right) \bar{u}_{j}, \\
\bar{S}=K^{-1} \bar{\Phi}=\sum_{j}^{N} \frac{\bar{u}_{j}^{T} \bar{\Phi}}{\lambda_{j}} \bar{v}_{j},
\end{gathered}
$$

where $(\bar{a}, \bar{b})$ denotes the usual scalar product of two vectors. We will show that in this form it is easier to appreciate where the ill-posed nature of the inverse problem resides.
The solution of the direct problem $\bar{\phi}$ is expressed on the basis set spanned by the left singular vectors $\bar{u}_{j}$ with coefficients that are proportional to the singular values and thus are damped exponentially fast as the index $j$ grows. In this sense, usually it is said that the integral transform is a smoothened version of the original signal.

On the contrary, in the inverse problem the coefficients of the expansion on the basis given by the right singular vectors $\bar{v}_{j}$ are exponentially amplified raising the index $j$. A necessary requirement in order to obtain anything meaningful out of it is that the overlaps $\bar{u}_{j}^{T} \bar{\phi}$ decay faster to zero than the singular values, and this is a really stringent property when the transform is computed with finite precision.

In practical situations, the singular-value decay is so fast that just the first few have a meaning because after a while the number gets completely corrupted by floating-point errors (see Fig. 1), and thus one expects meaningful results just for the first few terms in the sum [Eq. (14)]. This is a sign of the fact that when one discretizes an ill-posed problem, the corresponding discrete problem is numerically ill conditioned or, in other words, the problem is not stable with respect to uncertainties in the input.

The decay rate of the singular values towards zero can be used as an indication of the degree of ill-posedness of the problem: the faster the singular values go to zero, the worse is the impact of the ill-posed nature of the problem. This can be understood by initially noticing that for a $\delta$-function kernel all the singular values are one. When a function with a finite width is used, the SVD yields eigenvalues that start deviating more and more from this limit: the larger the width, the faster the decay. However, the SVD picks up not just information about the width, but also about the behavior of the function in the tails.

As an example, in Fig. 2 we compare the SVD of a Laplace kernel, two Lorentz kernels with different width, and a Sumudu kernel of a given width. The plot clearly shows that while the Sumudu kernel underperforms with respect to the
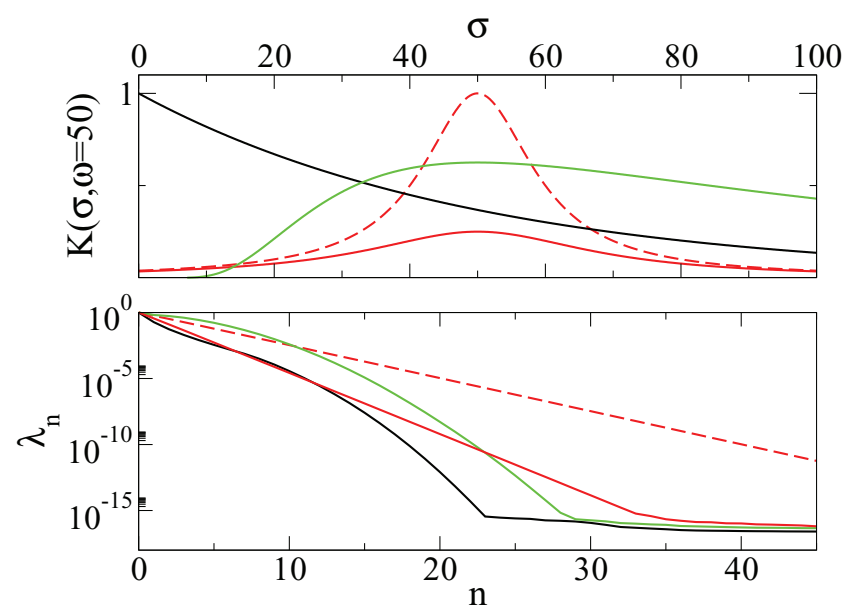

FIG. 2. (Color online) Upper panel: Comparison between Lorentz kernel (red curves) with width 20 (continuous line) and 10 (dashed line), a Sumudu kernel with $P=2$ and $b=2 a=2$ (solid green curve), and a Laplace kernel (solid black curve). Lower panel: The corresponding singular values for the kernels of the upper panel. 
Lorentz kernels, it widely overperforms the Laplace kernel. This is the key point of this paper: While it is not possible for technical reasons related to the nature of quantum Monte Carlo algorithms to use an optimal kernel form as a Guassian or a Lorentzian, which are good representations of a $\delta$ function with separately tunable center and width, we have found a kernel that, as discussed in the following, is (1) suitable for being used in projection QMC calculation, and (2) is a qualitatively and quantitatively better choice than a Laplace kernel. This prescription sets a more efficient way of analyzing QMC data in order to obtain information about the response function. Its application will result in an a priori milder ill-posedness of the inversion problem, which will in any case benefit from very efficient inversion schemes. The main advantage here is that almost no previous knowledge of the spectrum is required, and calculations can be predictive. However, there is an even more important point: the fact that the kernel $K_{P}(\sigma, \omega)$ tends to the $\delta$ function when $P \rightarrow \infty$, and Eq. (8) tells us that in principle if we could perform a calculation for very large $P$, we might get to the limit at which some information, such as the position of the peaks, has no need for any inversion to be extracted. This fact has been exploited, for example, in nuclear dynamics calculations. $^{22}$

\section{B. Quantum Monte Carlo}

As we mentioned, our aim is to extract dynamical information from projection QMC methods. These are all based on the well-known idea of filtering the ground state of a system from an initial ansatz by means of an imaginary-time propagation. This corresponds to solving the integral equation

$$
\Psi(R, \tau)=\int d R^{\prime} G\left(R, R^{\prime}, \tau\right) \Psi\left(R^{\prime}, 0\right) .
$$

The solution can be obtained, for instance, by sampling a spatial coordinate representation of the Green's function $G\left(R, R^{\prime}, \tau\right)=\left\langle R\left|e^{-\tau \hat{H}}\right| R^{\prime}\right\rangle$ to propagate a set of configurations representing in turn an expansion of the function $\Psi(R, \tau)$ in eigenstates of the position operator. This is the scheme usually employed in diffusion Monte Carlo calculations. Alternatively, it is possible to break up the Green's function in a product of short-time propagators in coordinate space:

$$
\begin{aligned}
\Psi(R, \tau)= & \int d R^{\prime} \ldots d R^{n} G\left(R, R^{n}, \Delta \tau\right) \\
& \times G\left(R^{n}, R^{n-1}, \Delta \tau\right) \ldots G\left(R^{\prime \prime}, R^{\prime}, \Delta \tau\right) \psi\left(R^{\prime}, 0\right),
\end{aligned}
$$

with $\tau=n \Delta \tau$. This formulation is implemented in the so-called path-integral ground-state methods ${ }^{23}$ and in the reptation Monte Carlo (RMC) algorithm, ${ }^{24}$ where the whole path $\left\{R, R^{\prime}, R^{\prime \prime}, \ldots, R^{n}\right\}$ is sampled from the product of the short-time propagators $G$, possibly modified with the use of a suitable importance function $\Psi_{T}$ to be determined in a variational calculation. The fact that estimating $\Phi(\mathbf{q}, \sigma)$ for the particular choice (7) for the integral kernel reduces to the computation of an imaginary-time correlation function makes this second formulation more convenient and straightforward. In particular, path-based methods yield estimates that never depend on the (necessary) importance function used to improve the convergence of the calculation.

In order to evaluate the transform (3) with the kernel function (7) within a QMC approach, we need to compute the imaginary-time correlation function, and then construct the corresponding linear combinations.

A small width of the kernel (and thus a less ill-posed problem to solve) might be achieved either using a large value of $P$ or by reducing the value of the ratio $b / a$. In both cases, this would require the computation of the imaginary-time correlation function for long imaginary time. This might indeed be a serious complication. However, a kernel that has a width smaller or comparable to the distance between the typical structures of the DSF is in principle sufficient to extract useful information from the transform. We have also confirmed this in numerical tests. In the application described in the following, we used the kernel $\sigma K_{P}(\sigma, \omega)$ with the typical values of $P=2, a=1$, and $b=2$.

\section{APPLICATION TO LIQUID HELIUM}

As a first benchmark application to a realistic physical problem, we have considered the density excitation response in bulk atomic ${ }^{4} \mathrm{He}$ at $T=0$. The system is modeled as a box with periodic boundary conditions, containing $N=64$ or 125 ${ }^{4} \mathrm{He}$ atoms interacting via the HFDHE2 pairwise potential, ${ }^{25,26}$ which quantitatively reproduces the binding energy of bulk ${ }^{4} \mathrm{He}$ up to the freezing point, effectively including three-body contributions. More accurate two-body interactions give an overbinding of the system and need to be complemented by a three-body force. Explicit three-body interactions can be included in a quantum Monte Carlo calculation, but the computational cost becomes much larger. Calculations are performed at the experimental saturation density $\left(n_{0}=\right.$ $0.02186 \AA^{-3}$ ). The density excitation operator is defined as

$$
\hat{O}(\mathbf{q}) \equiv \hat{\rho}=\sum_{i=1}^{N} e^{i \mathbf{q} \cdot \mathbf{r}_{i}}
$$

and the transformed DSF in Eq. (3) becomes

$$
\Phi(\mathbf{q}, \sigma)=\left\langle\Psi_{0}\left|\sum_{i, j=1}^{N} e^{i \mathbf{q} \cdot \mathbf{r}_{i}} K_{P}(\sigma, \hat{H}) e^{-i \mathbf{q} \cdot \mathbf{r}_{j}}\right| \Psi_{0}\right\rangle .
$$

As it is customary in neutron spectroscopy, one can distinguish the contribution coming from the so-called coherent part, given by the terms with $i \neq j$, related to collective excitations, and an incoherent part with $i=j$ that essentially picks up contributions from single-particle excitations. We have obtained results for both the full and for its incoherent parts, in the most studied region of the spectrum: the low-momentum phonon-maxon-roton part $q \approx 0.3 \div 2.8 \AA^{-1}$. Computations have been performed by means of a reptation Monte Carlo (RMC) algorithm, as described in Ref. 24. The variational importance function includes two- and three-body correlations expanded in a basis $\operatorname{set}^{27}$ and optimized using a variational Monte Carlo procedure. Ground-state properties are well reproduced: the ground-state energy per particle is $\epsilon_{0}^{\mathrm{RMC}}=$ $-7.23 \pm 0.01 \mathrm{~K}$, in good agreement with previous calculations using the same potential, ${ }^{24}$ and with the experimental value 
$\epsilon_{0}^{\text {expt }}=-7.17 \mathrm{~K}$. The static structure factor $S(\mathbf{q})$ is consistent with experimental data and previous calculations. ${ }^{24,27}$

Turning to the result on $S(\mathbf{q}, \omega)$, the striking difference between the estimate obtained inverting the transform with the Laplace kernel or the one in Eq. (5) can be seen in Fig. 5, where we compare the results of the inversion obtained from RMC data with both kernels [in the figure $S(q, \omega)$ has been normalized to the static structure factor $S(q)$ ]. Apart from the small shift of the peak due to the $0.04-\AA^{-1}$ difference in the momentum transfer (the momenta are limited by the discretization imposed by the use of a finite simulation cell, here $L=14.306 \AA$ ), the new kernel permits us to retrieve the information on the second peak and gives a much more realistic height and width of the one-phonon peak. It should be noted that at $T=0$ the peak corresponding to the collective excitation should be substantially narrower than the measured one at higher $T$. An estimate of the intrinsic peak width is $\Delta \omega \simeq 5 \times 10^{-4} \mathrm{~K}^{28}$ Therefore, the experimental width is essentially due to the resolution of the apparatus. ${ }^{29}$

The power of the new kernel appears to be clear from the inset in Fig. 3, where the two transforms obtained with the Laplace kernel $\left[K(\sigma, \omega)=e^{-\omega / \sigma}\right]$ and with the kernel in (5) are shown. In the former case, no structure is visible, while a clear signature of a resonance is visible in the latter case in the interesting region. This is due to the fact that the new kernel is a representation of the delta function. Even if we have used $P=2$ and therefore its width is rather large around the first resonance energy (see Fig. 4), the inversion procedure is able to recover a narrow peak. In addition, a second peak is recovered, even if the width of the kernel is larger in that region.

Above we stated that our main point is on the improvement that one can attain from a wise choice of the integral transform to invert, and not on the particular inversion scheme used. Nonetheless, a few comments are necessary here concerning the methods used to invert the transform. We have used three different methods: the entropy maximization maximum likelihood (EMML) ${ }^{30}$ the simultaneous algebraic reconstruction technique $^{30}$ (SMART) (both with the unique constraint of a

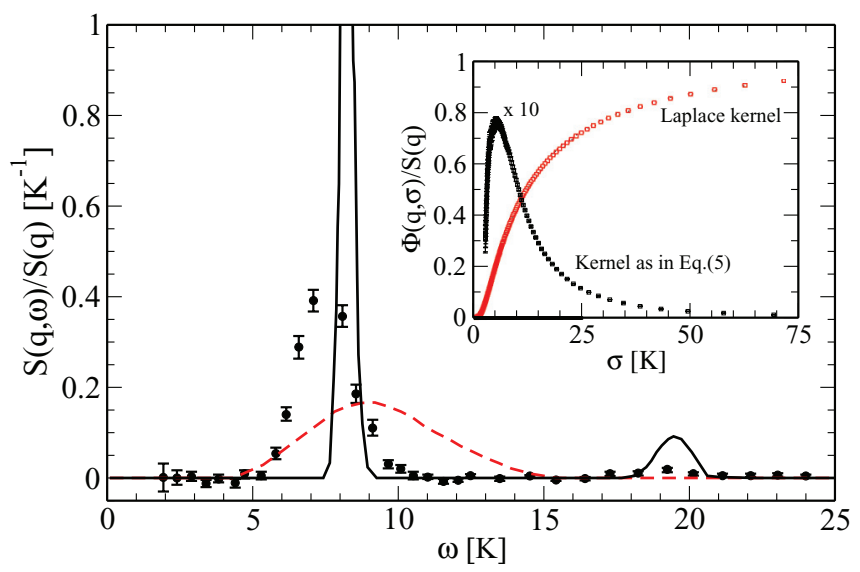

FIG. 3. (Color online) $\mathrm{S}(q, \omega)$ in liquid ${ }^{4} \mathrm{He}$ at $T=0$ and $q=$ $0.44 \AA^{-1}$. Black solid curve: inversion of IT with kernel in Eq. (5). Red dashed curve: inversion of IT with Laplace kernel. Experimental data (black dots) are from Ref. 29 (at $T=1.34 \mathrm{~K}$ and $q=0.4 \AA^{-1}$ ). In the inset, the corresponding ITs are shown.

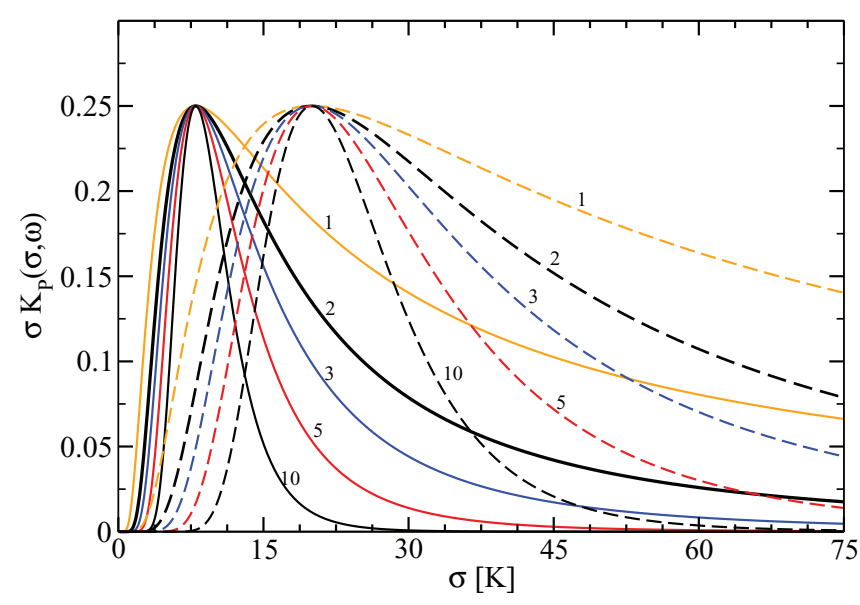

FIG. 4. (Color online) Plot of $K_{P}(\sigma, \omega=8 \mathrm{~K})$ (full lines) and $K_{P}(\sigma, \omega=20 \mathrm{~K})$ (dashed lines), for different values of $P$. All curves have been normalized to the maximum value of $\sigma K_{1}(\sigma, \omega)$.

positive definite DSF), and a simpler regularization method. The latter consists in writing

$$
S(\mathbf{q}, \omega)=\sum_{m}^{M} c_{m} \frac{1}{\sqrt{2 \pi} \Gamma_{m}} e^{-\left(\frac{\omega-\omega_{m}}{\sqrt{2} \Gamma_{m}}\right)^{2}},
$$

evaluating its IT and performing a (nonlinear) best fit of the parameters $c_{m}, \omega_{m}$, and $\Gamma_{m}$ on $\Phi(\sigma)$ calculated by RMC for a large a large number of $\sigma$ values (300 points). In Fig. 5, the results obtained with the different inversions are shown. One notices that the first narrow peak is rather stable against the three methods. A second peak is also predicted in all cases. Its position and width is very stable against the inversion with EMML or SMART. As expected, the fit with the ansatz in (18) is too rough to be reliable in a region where the kernel has too large a width, as shown in Fig. 4.

In Fig. 6(a), we have plotted the dispersions of the collective modes obtained using the new transform. The bars in the figures indicate the computed widths of the excitations. Both the peak positions and the linewidths are robust with respect to

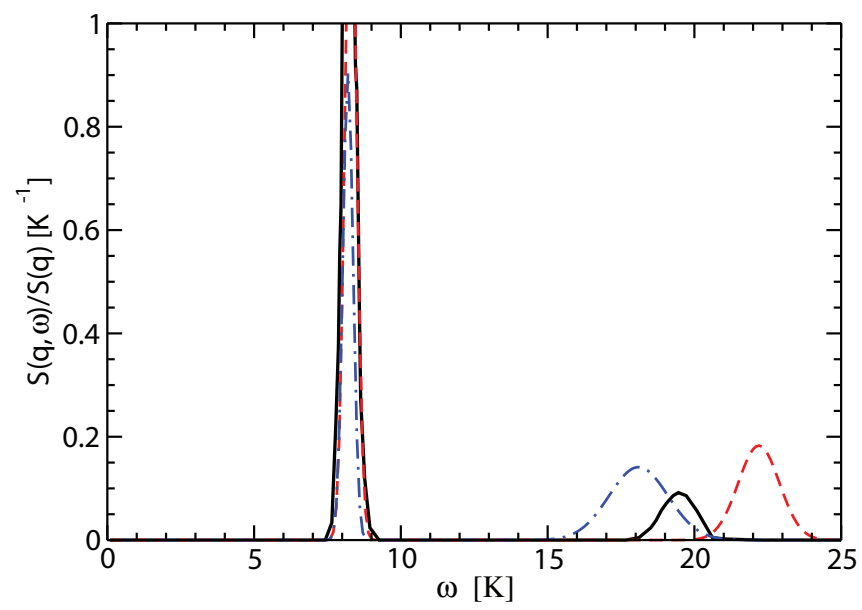

FIG. 5. (Color online) $\mathrm{S}(q, \omega)$ in liquid ${ }^{4} \mathrm{He}$ at $T=0$ and $q=$ $0.44 \AA^{-1}$. Inversion with SMART or EMML (solid black line), inversion with ansatz in Eq. (18) and $M=2$ (dashed red line) and $M=3$ (dashed-dotted blue line). 


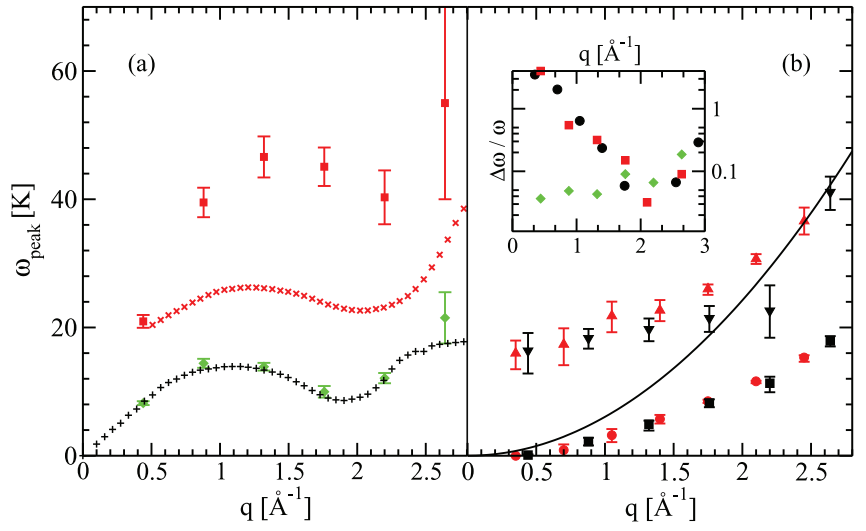

FIG. 6. (Color online) (a) Dispersion of the collective modes in liquid ${ }^{4} \mathrm{He}$ at equilibrium density and $T=0$. Computed values for the first (green diamonds) and second (red squares) peaks. Error bars are estimates of the widths of the peaks. + and $\times$ are the corresponding experimental data from Ref. 31 at $T=1.1 \mathrm{~K}$. (b) Dispersion of the first peak (red circles: box with $N=64$; black squares: box with $N=125$ ) and second peak (red up triangles: box with $N=64$; black down triangles: box with $N=125$ ) of the incoherent DSF. Full line: free-particle excitation spectrum. (Inset) half-widths of the first peak for both incoherent excitations (black circles: box with $N=64$; red squares: box with $N=125$ ) and coherent ones (green diamonds: box with $N=64$ ).

EMML or SMART inversions. We found that for $q \leqslant 2.4 \AA^{-1}$, methods converge to the same solutions.

The experimental low-lying part ${ }^{29}$ is extremely well reproduced up to $q \approx 2.6 \AA^{-1}$. The two-phonon branch is clearly visible and well resolved. As it happens in Ref. 7, it only qualitatively compares to the experiment. The calculated widths of the second-branch excitations are much smaller than what is obtained from experiments. At present, it is difficult to judge the reason for this discrepancy. It can be ascribed to temperature effects and inadequacy of the Aziz pair potential to describe multiexcitation processes. It might be as well an indication that the width of our kernel is not small enough in that energy region to allow a more robust estimation.

The results in Fig. 6(a) were obtained combining two simulations at $\Delta \tau=0.002$ and $0.001 \mathrm{~K}^{-1}$, respectively, obtaining a mesh separation less than 0.5 until about $40 \mathrm{~K}$. In order to obtain meaningful results in the high-energy regime, a large collection of RQMC data taken with different imaginary-time steps is needed in order to increase the sampling points. Due to this technical difficulty, at present we have not performed an exhaustive research in the high-momentum-transfer limit. However, preliminary calculations show that the spectrum has the expected approximately free-particle-like behavior, and that for $q \approx 6 \AA^{-1}$ and above the incoherent part of the DSF accounts for the total scattering.

Indeed, a useful feature of these calculations is that one can separately compute the incoherent part of the full response function, in order to help interpreting the result in terms of possible single-particle and collective excitations.

In Fig. 6(b), we have plotted the calculated excitation spectrum of single-particle excited states. The spectrum shows at least two distinct branches. A lower-energy excitation starts from $q \approx 0.5 \AA^{-1}$ and propagates with a velocity of the same order of magnitude as the superfluid critical velocity. A second branch can be observed starting at an energy slightly below two times the roton energy, tending asymptotically to the free-particle spectrum. In order to help the interpretation of these peaks as single-particle excitations, in the inset of Fig. 6(b) we show the relative half-width in energy of these peaks as compared to that of the lower branch in the full spectrum [Fig. 6(a)]. We see that for low-momentum transfer, the dominant long-living excitations are the coherent ones, while as we approach the roton minimum the incoherent contributions start playing an important role.

Interestingly enough, the lower-energy branch crosses the collective excitation spectrum exactly at the roton minimum, thereby reinforcing the picture of the roton as a single-particle excitation of an atom exiting the superfluid. The behavior of these single-particle excitations might be significantly affected by the quantum many-body correlations induced by the particle-particle interaction.

At intermediate values of the momentum $q \geqslant 2.8 \AA^{-1}$, the positions of the peaks become less stable with respect to the specific inversion procedure employed. This might be a signature of the fact that our resolution in that regime is too low.

In order to illustrate this fact, we report in Fig. 7 a typical result obtained at high-momentum transfer. We compare our results with experiments obtained at nonzero pressure and temperature from Ref. 32. The experiment presents a well-distinct peak along with a slowly decaying tail (generally interpreted as due to single-particle excitations ${ }^{32,33}$ ). The theoretical curve shows instead a single peak in a position that is not very far from the overall centroid of the experimental response. In this case, the existence of a collective peak can not be established separately. In the graph, a typical $\sigma K_{P}(\sigma, \omega)$ (in the specific case $\omega=18 \mathrm{~K}$ ) is also displayed. We point out once more that for a given value of $P$ the kernel function becomes broader when increasing $\sigma$. In this case, it is evident that the width makes the kernel qualitatively similar to the Laplace one.

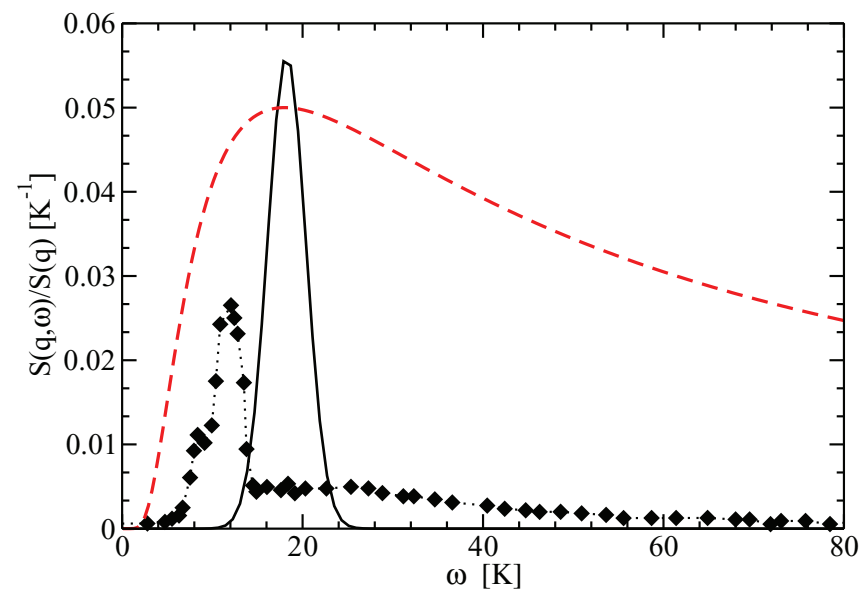

FIG. 7. (Color online) $S(q, \omega)$ for liquid ${ }^{4} \mathrm{He}$ at $T=0 \mathrm{~K}$ and $q=2.64 \AA^{-1}$ (solid line), compared with the results of Ref. 32 for $q=2.4 \AA^{-1}, T=0.6 \mathrm{~K}$, and at pressure $20 \mathrm{bar}$ (dots). The red curve (in arbitrary units) is an example of $\sigma K_{P}(\sigma, \omega)$ used for computing the inverse response function in this energy range. Notice that the last curve is plotted as a function of $\sigma$ (in $\mathrm{K}$ ) with a fixed value of $\omega=18 \mathrm{~K}$. 
The computational cost required to use a narrower kernel (corresponding to a larger value of $P$ ) which would allow us to resolve the structures observed at large $q$ dramatically increases due to the need of using longer imaginary-time paths. This is the current limit of applicability of the method when used along with unsophisticated inversion techniques.

\section{SUMMARY}

We have proposed an IT method to extract, with a controlled resolution, excitation spectra from numerical calculations that implement imaginary-time propagation of an initial state, such as DMC or RMC. We remark that the aim of this work is to show that there is a complementary way to improve our ability to obtain dynamical information from Monte Carlo simulation, aside from the mere improvement of the inversion algorithm itself: a wise choice of the integral kernel. In this respect, the most important result is to have found a kernel, appropriate to QMC calculations, which is a representation of the $\delta$ function, and therefore allows a reliable inversion of the transform with a high degree of predictability.

The application to the study of the excitations in ${ }^{4} \mathrm{He}$ shows the robustness and the higher resolution power attainable with this kernel, even with the rather simple regularization techniques used. In fact, at $T=0$ an $a b$ initio calculation with the unique ingredient of a potential has allowed us to to predict with a high degree of reliability the existence of a very narrow peak at an energy of about $8 \mathrm{~K}$ for $q=0.44 \AA^{-1}$ and of a second peak at higher energy. The method has allowed us to calculate coherent and incoherent contributions separately, helping the interpretation of the spectrum in terms of collective and single-particle phenomena.

Computations might be easily extended to the $T \neq 0$ case by using standard path-integral Monte Carlo methods and a slightly modified version of the kernel function (5). The limit to the accuracy of the spectra is in principle only the available computer power.

\section{ACKNOWLEDGMENTS}

We thank W. Leidemann, V. Efros, G. V. Chester, M. H. Kalos, J. Carlson, and S. Gandolfi for useful and stimulating discussions about this subject. Calculations have been partially carried out on the supercomputer AURORA in the framework of the AURORA-Science project funded by INFN and the Bruno Kessler Foundation (FBK). Authors are members of LISC, Interdisciplinary Laboratory for Computational Science, a joint venture of FBK and the University of Trento. *roggero@science.unitn.it

†pederiva@science.unitn.it

†orlandin@science.unitn.it

${ }^{1} \mathrm{~J}$. Hadamard, Lectures on Cauchy's Problem in Linear Partial Differential Equations (Dover, New York, 1952).

${ }^{2}$ A. Tikhonov and V. Arsenin, Solutions of Ill-posed Problems (Wiley, New York, 1977).

${ }^{3}$ A. Talbot, J. Inst. Math. Applicat. 23, 97 (1979).

${ }^{4}$ M. Boninsegni and D. M. Ceperley, J. Low Temp. Phys. 104, 339 (1996).

${ }^{5}$ E. T. Jaynes, in Statistical Physics, edited by K. Ford (Benjamin, New York, 1963), p. 181.

${ }^{6}$ R. N. Silver, D. S. Sivia, and J. E. Gubernatis, Phys. Rev. B 41, 2380 (1990).

${ }^{7}$ E. Vitali, M. Rossi, L. Reatto, and D. E. Galli, Phys. Rev B 82, 174510 (2010).

${ }^{8}$ S. R. White, Computer Simulation Studies in Condensed Matter Physics III (Springer, Berlin, 1991), pp. 145-153.

${ }^{9}$ A. W. Sandvik, Phys. Rev B 57, 10287 (1998).

${ }^{10}$ V. D. Efros, W. Leidemann, and G. Orlandini, Phys. Lett. B 338, 130 (1994).

${ }^{11}$ V. D. Efros, W. Leidemann, G. Orlandini, and N. Barnea, J. Phys. G: Nucl. Part. Phys. 34, R459 (2007).

${ }^{12}$ S. Bacca, M. A. Marchisio, N. Barnea, W. Leidemann, and G. Orlandini, Phys. Rev. Lett. 89, 052502 (2002).

${ }^{13} \mathrm{~S}$. Bacca, H. Arenhoevel, N. Barnea, W. Leidemann, and G. Orlandini, Phys. Lett. B 603, 159 (2004).

${ }^{14}$ V. D. Efros, Yad. Fiz. 41, 1498 (1985) [Sov. J. Nucl. Phys. 41, 949 (1985)].

${ }^{15}$ V. D. Efros, W. Leidemann, and G. Orlandini, Few-Body Syst. 14, 151 (1993).
${ }^{16}$ T. Munehisa and Y. Munehisa, arXiv:cond-mat/0001304.

${ }^{17}$ P. E. Kornilovitch, arXiv:cond-mat/0010100.

${ }^{18}$ R. A. Bertlmann, G. Launer, and E. de Rafael, Nucl. Phys. B 250, 61 (1985)

${ }^{19}$ G. Orlandini, T. Steele, and D. Harnett, Nucl. Phys. A 686, 261 (2001).

${ }^{20}$ G. Orlandini, W. Leidemann, V. D. Efros, and N. Barnea, J. Phys. G: Nucl. Part. Phys. 37, 064011 (2010).

${ }^{21}$ G. K. Watugala, Int. J. Math. Ed. Sci. Technol. 24, 35 (1993).

${ }^{22}$ S. Bacca, N. Barnea, G. Hagen, G. Orlandini, and T. Papenbrock, arXiv:1303.7446.

${ }^{23}$ A. Sarsa, K. E. Schmidt, and W. R. Magro, J. Chem. Phys. 113, 1366 (2000).

${ }^{24}$ S. Baroni and S. Moroni, Phys. Rev. Lett. 82, 4745 (1999); arXiv:cond-mat/9808213.

${ }^{25}$ M. H. Kalos, M. A. Lee, P. A. Whitlock, and G. V. Chester, Phys. Rev. B 24, 115 (1981).

${ }^{26}$ R. A. Aziz, J. S. Carley, V. P. S. Nain, W. L. Taylor, and G. T. McConville, J. Chem. Phys. 70, 4330 (1979).

${ }^{27}$ S. Moroni, S. Fantoni, and G. Senatore, Phys. Rev. B 52, 13547 (1995).

${ }^{28}$ F. Mezei and G. W. Stirling, in 75th Jubilee Conference on Helium4, edited by J. G. M. Armitage (World Scientific, Singapore, 1983), p.111.

${ }^{29}$ W. G. Stirling and H. R. Glyde, Phys. Rev. B 41, 4224 (1990).

${ }^{30}$ C. L. Byrne, IEEE Trans. Image Process. IP-2, 96 (1993).

${ }^{31}$ A. Woods and R. Cowley, Can. J. Phys. 49, 177 (1971).

${ }^{32}$ J. V. Pearce, R. T. Azuah, B. Fak, A. R. Sakhel, H. R. Glyde, and W. Stirling, J. Phys.: Condens. Matter 13, 4421 (2001).

${ }^{33}$ B. Fåk, L. P. Regnault, and J. Bossy, J. Low Temp. Phys. 89, 345 (1992). 